\title{
Electrospun Antimicrobial Polyurethane Nanofibers Containing Silver Nanoparticles for Biotechnological Applications
}

\author{
Faheem A. Sheikh \\ Department of Bionano System Engineering, Chonbuk National University, Jeonju 561-756, Korea \\ Nasser A. M. Barakat* \\ Chemical Engineering Department, Faculty of Engineering, El-Minia University, El-Minia, Egypt \\ Center for Healthcare Technology Development, Chonbuk National University, Jeonju 561-756, Korea \\ Muzafar A. Kanjwal \\ Department of Polymer Nano Science and Technology, Chonbuk National University, Jeonju 561-756, Korea
}

Atul A. Chaudhari, In-Hee Jung, and John Hwa Lee

College of Veterinary Medicine, Chonbuk National University, Jeonju 561-756, Korea

Hak Yong Kim*

Department of Textile Engineering, Chonbuk National University, Jeonju 561-756, Korea

Received November 27, 2008; Revised February 7, 2009; Accepted February 8, 2009

\begin{abstract}
In this study, a new class of polyurethane (PU) nanofibers containing silver (Ag) nanoparticles (NPs) was synthesized by electrospinning. A simple method that did not depending on additional foreign chemicals was used to self synthesize the silver NPs in/on PU nanofibers. The synthesis of silver NPs was carried out by exploiting the reduction ability of $N, N$-dimethylformamide (DMF), which is used mainly to decompose silver nitrate to silver NPs. Typically, a sol-gel consisting of $\mathrm{AgNO}_{3} / \mathrm{PU}$ was electrospun and aged for one week. Silver NPs were created in/on PU nanofibers. SEM confirmed the well oriented nanofibers and good dispersion of pure silver NPs. TEM indicated that the Ag NPs were 5 to $20 \mathrm{~nm}$ in diameter. XRD demonstrated the good crystalline features of silver metal. The mechanical properties of the nanofiber mats showed improvement with increasing silver NPs content. The fixedness of the silver NPs obtained on PU nanofibers was examined by harsh successive washing of the as-prepared mats using a large amount of water. The results confirmed the good stability of the synthesized nanofiber mats. Two model organisms, E. coli and S. typhimurium, were used to check the antimicrobial influence of these nanofiber mats. Subsequently, antimicrobial tests indicated that the prepared nanofibers have a high bactericidal effect. Accordingly, these results highlight the potential use of these nanofiber mats as antimicrobial agents.
\end{abstract}

Keywords: electrospinning, nanofibers, silver nanoparticles, antimicrobial, zones of inhibition, morphological changes.

\section{Introduction}

Formation of super-bugs has become a main problem due to frequent use of the antibiotics. Generally, due to genetic transformation of microbial strains had created resistance against the present antibiotics. ${ }^{1}$ In order to have complete removal of pathogenic strains during wound healing process, there is a need to have an alternate strategy. Silver (Ag)

\footnotetext{
*Corresponding Authors. E-mails: khy@chonbuk.ac.kr or nasbarakat@yahoo.com
}

is a powerful natural antibiotic being used since ancient times for the purpose of wound healing. When silver comes in contact with microorganisms, it leads to sudden distortion of cell wall which later on causes death of these organisms, therefore, progressive steps are made for future use of the silver based materials. ${ }^{2}$ Silver is considered to be active against multiple drug resistant microbial strains. Surprisingly, there are minimal chances for development of bacterial resistance due to immediate death of microorganisms upon contact with silver ions. The mechanism for development of minimal resistance against silver has been well doc- 
umented. ${ }^{3}$ This ability to create minimal or no resistance in microorganisms can lead them to replace impotent antibiotics. ${ }^{4}$ Beside its antimicrobial activity, it has been found that the use of silver based dressing materials enhances epithelialization in clean wounds of animal model which indicates synergistic effect of silver ions as antimicrobials and as well as wound healing agents. ${ }^{5}$ Modification of silver and its compounds is important to be applicable in various medical and paramedical fields which is quite challenging for scientists till now. It is well known that the surface area of the inorganic antibiotics is a main parameter, consequently, formulation of $\mathrm{Ag}$ in nanostructural form is expected to have interesting behavior. However, utilizing these nanostructural forms is not an easy task. Supporting of Ag nanoparticles (NPs) on polymeric matrix is a unique and effective methodology being exploited by many researchers. Therefore, many studies had been carried out in this concern to produce Ag based materials applicable in various applications such as an antimicrobial filters, ${ }^{6}$ wound dressing materials, ${ }^{7}$ water disinfectants, ${ }^{8}$ chemical sensors, ${ }^{9}$ and protective cloths. ${ }^{10,11}$

Actually, not only the antimicrobial agents (i.e. silver) do have solitary importance but also the polymer matrix also plays a vital role, so, many polymers have been exploited. Generally, for the purpose of using a polymer in antimicrobial or wound healing materials, it should have the following characters: water insolubility, appropriate pore size, non invasive to human cells so as to support the epithelialization. Recently, many polymers have been utilized; like cellulose acetate, ${ }^{12}$ poly(acrylonitrile) ${ }^{13}$ poly(caprolactone),${ }^{14}$ poly(methyl methacrylate) ${ }_{15}^{15}$ poly(vinyl alcohol) ${ }^{16}$ and polyimide fibers. ${ }^{17}$ Unfortunately, all the reports in such a field have focused only on the synthesizing procedure and ignored some important parameters affecting utilizing the final products in nano-biotechnological applications, for instance fixedness of the obtained silver nanoparticles and analyses of these nanofibers for antimicrobial assay. Therefore, preparing of silver NPs/polymer nanofiber matrix with taken in consideration all the aforementioned parameters was the aim of the present study.

During the past few decades, electrospinning technique has been paid a considerable attention due to production of fibers having diameter in the range of few microns to nanometer scale by applying high electric fields. ${ }^{18,19}$ In a typical electrospinning process, electrostatically driven polymer jet is ejected from polymer solution which undergoes bending instability wherein the solvent evaporates and ultra fine stretched fibers are deposited on the grounded collector. ${ }^{20}$ Nanofibers received from this technique have been drawn attention due to their web like nature which exactly mimic the topology of extracellular matrix present in human body therefore, are used as scaffolds in tissue engineering. ${ }^{21}$ Nanostructured materials especially, nanofibers prepared via electrospinning of biocompatible polymer materials have tremendous abilities such as a high surface to volume ratio and high porosity which can be used as supporting aid for filter membranes for water purifying systems. ${ }^{22}$

Polyurethane (PU) is thermoplastic polymer having excellent mechanical properties ${ }^{23}$ and water insolubility. ${ }^{24}$ Moreover, it can be used as biomaterials. Recently, it has been explored that PU nanofibers have tremendous applications in various fields; biosensors, ${ }^{25}$ protective cloths ${ }^{26}$ and enhancing epithelial growth. ${ }^{27}$ There are various articles regarding electrospinning of modified PU to be used as antimicrobial fibers. ${ }^{28,29}$ However, according to best of our knowledge, there is no report dealing with producing PU nanofibers/silver NPs to exploit their prompt features.

In the present study PU nanofibers containing Ag NPs polymeric matrix has been successfully produced by using the electrospinning technique without adding any foreign reducing agent. Moreover, evaluation of the obtained nanofiber matrices for morphological properties and crystalline structure were investigated. Stability of the produced silver NPs/PU nanofiber mats has been studied by several successive washing processes. Antimicrobial activities for two gram negative test microorganisms have been evaluated. According to the obtained results, one can say that the prepared silver NPs/PU nanofibrous matrix could be properly employed as recommended candidate for many biological applications such as internal aid for water and air filters membranes and for prolonged antimicrobial wound dressing agents.

\section{Experimental}

Materials. Polyurethane (PU, MW=110,000, medical grade) and silver nitrate $\left(\mathrm{AgNO}_{3}\right)$ were purchased from Cardio Tech. Intern., Japan, and Junsei Chemical Ltd., Japan, respectively. Tetrahydrofuran (THF) and $N, N$-dimethylformamide (DMF) (analytical grade, Showa Chemicals Ltd., Japan) were used as solvents without further purification. Difco Mueller Hinton Agar and Standard Plate Count Agar were purchased from Becton, Dickinson \& Co., (Spark, MD 221 Ltd., USA) and Hampshire, (Oxoid Ltd. England). Phosphate buffer saline (PBS) 0.1 M, pH 7.4 was purchased from (Aldrich Co., USA). For checking antimicrobial activity two microbial strains as E. coli (ATCC 43890) and S. typhimurium obtained from (National Veterinary Quarantine service Korea) were used as a model organisms to check antimicrobial activity.

Characterization. The morphology of the nanofiber mats had been analyzed by JEOL JSM-5900 scanning electron microscope, JEOL Ltd., Japan. Information about the phase and crystallinity was obtained by using Rigaku X-ray diffractometer (XRD, Rigaku Co., Japan) with $\mathrm{Cu} \mathrm{K} \alpha(\lambda=$ $1.540 \AA$ ) radiation over Bragg angle ranging from $30^{\circ}$ to $80^{\circ}$. Transmission electron microscopy (TEM) was done by JEOL JEM 2010 (TEM) operating at $200 \mathrm{kV}$, JEOL Ltd., Japan. The mechanical properties of the nanofiber mats were investigated by universal testing machine (UTM, AG- 
5000G, Shimadzu Co., Japan) under a crosshead speed of 5 $\mathrm{mm} / \mathrm{min}$. Samples were prepared in the form of standard dumbbell shaped according to guidelines of ASTM standard via die cutting from nonwoven mats and tested in the machine direction at least five specimens were tested for each sample. UV-Visible measurements were performed by using HP 8453 UV-Visible spectroscopy system (Germany), the absorbance was measured from 400 to $1,100 \mathrm{~nm}$; wavelength. The spectra obtained were analysed by HP ChemiStation software 5890 series. Average pore diameter, pore volume, pore area, and total porosity of the electrospun membrane were measured by the Auto Pore IV 9500 V1.05 mercury porosimeter (Micrometritis Instrument, Co., Germany). The pre-weighed rectangular shaped electrospun membranes of average $0.25 \mathrm{~mm}$ thickness were cut into $1 \times 3$ $\mathrm{cm}^{2}$ as sample for analyses. Sample was placed in the cup of the penetrometer ( $\mathrm{s} / \mathrm{n}$-(10) 5 Bulb, 1.131 stem power), which was closed by tightening the cap. Mercury filling pressure was $3.4 \mathrm{kPa}(0.42 \mathrm{psia})$ with evacuation time of $5 \mathrm{~min}$. To find out the effect of silver NPs, on bacterial morphology; nanofibrous membranes were examined by Bio-SEM (Hitachi Co., Japan).

Procedure.

Fabrication of Nanofibers by Electrospinning: Pure PU $10 \mathrm{wt} \%$ was prepared by stepwise dissolving in THF and DMF. Initially PU pellets were overnight dissolved in THF after that DMF was added to produce final sol-gel containing $10 \mathrm{wt} \%$ of PU in THF/DMF $(1: 1, \mathrm{w} / \mathrm{w}) . \mathrm{AgNO}_{3} / \mathrm{DMF}$ solutions were prepared and added to the PU sol-gel to have final mixtures containing silver nitrate of 2, 5, 7, $10 \mathrm{wt} \%$; with respect to the polymer concentration. A care was taken to protect the samples from light while working with $\mathrm{AgNO}_{3}$. A high voltage power supply (CPS-60 K02V1, Chungpa EMT Co., Republic of Korea), capable of generating voltages up to $60 \mathrm{kV}$, was used as a source of electric field for spinning of nanofibers. Polymer solution to be electrospun was supplied through a glass syringe attached to a capillary tip. The copper wire originating from positive electrode (anode) connected with graphite pin was inserted into the polymer solution and a negative electrode (cathode) was attached to a metallic collector. Briefly, the solutions were electrospun at $20 \mathrm{kV}$ voltage and $15 \mathrm{~cm}$ working distance (the distance between the needle tip and the collector). The as-spun fibers were stored for 1 week then vacuously dried for $24 \mathrm{~h}$ to remove the residual solvents.

Zone of Inhibition: Analysis of zones of inhibition (ZoI) was carried out by making samples from silver-free PU nanofiber mats and silver containing ones, the samples were cut into round disk shape by cork borer having diameter of $13 \mathrm{~mm}$. Before using, the samples were sterilized by ethylene oxide (EO). The microbial population were efficiently raised and spectroscopically checked to reach density of $1 \times 10^{8}$ cells $/ \mathrm{mL}$ by McFarland method. The test has been carried out as follow, a cell suspension of $1 \times 10^{8}$ cells $/ \mathrm{mL}$ was inoculated over a plate containing solidified nutrient agar medium onto disposable sterilized petriplates by spread plate method. Later on, the sterilized nanofiber samples were gently placed over the solidified agar gel under aseptic conditions. Plates were incubated for $12 \mathrm{~h}$ at $37^{\circ} \mathrm{C}$, and then observation of the zones of inhibition was investigated.

Cell Morphology Changes: Cell suspensions of E. coli and S. typhimurium having density of $1 \times 10^{8}$ cells $/ \mathrm{mL}$ in PBS were prepared. Pre-weighed electrospun membranes of 0.25 $\mathrm{mm}$ thickness were cut into $1 \times 3 \mathrm{~cm}^{2}$ of rectangular shapes were immersed in $1 \times 10^{8}$ cells $/ \mathrm{mL}$ in PBS solutions in shaking incubator at $37^{\circ} \mathrm{C}$ for a period of $4 \mathrm{~h}$. After such time period; samples were taken out form the microbial population. The bacterial fixation on nanofiber mats was conducted as follow: Nanofiber disks obtained after immersion in $1 \times 10^{8}$ cells $/ \mathrm{mL}$ in PBS solutions were washed with fresh PBS in a six well plate to remove the excess of bacteria, PBS drain was removed. The remaining cells on the nanofibers were well fixed on the nanofiber mats by adding a proper amount of PBS containing 3\% glutaraldehyde, and then the six well plate containing the samples immersed in PBS/glutaraldehyde solution were stored at $4{ }^{\circ} \mathrm{C}$ for $5 \mathrm{~h}$. After such time, the nanofiber mats were removed from the solution and washed with PBS followed by dehydration steps via $25,50,75$, and $100 \%$ ethanol for 15 min each. Further on, the excess of ethanol was removed from the nanofiber mats by overnight vacuum drying, and then the samples were investigated by Bio-SEM.

Colony Forming Units: Bacterial cells of $E$. coli and $S$. typhimurium were resuspended to provide a final density of $1 \times 10^{8} \mathrm{CFU} / \mathrm{mL}$ in PBS according to $0.5 \mathrm{McF}$ arland turbidity standard (approximately 1 to $2 \times 10^{8} \mathrm{CFU} / \mathrm{mL}$ ). In order to obtain countable colony number, it is noteworthy mentioning that 10 -fold dilutions were performed to obtain the final density of $1 \times 10^{7} \mathrm{CFU} / \mathrm{mL}$ in nutrient broth. Disks $(13 \times 13 \mathrm{~mm})$ from silver-free and silver containing PU nanofiber mats were sterilized with EO gas for $30 \mathrm{~min}$, and then immersed in $5 \mathrm{~mL}$ of the bacterial suspension in a $50 \mathrm{~mL}$ conical tube, the media were shaken at $200 \mathrm{rpm}$ at $37^{\circ} \mathrm{C}$ for $30,60,90$ and 120 min each. After shaking incubation, $100 \mu \mathrm{L}$ of culture media was spread over entire agar surface. Thereafter plates were incubated at $37{ }^{\circ} \mathrm{C}$ for overnight and $\mathrm{CFU} / \mathrm{mL}$ was counted.

\section{Results and Discussion}

Previous works have indicated that DMF does have the ability to reduce some metallic precursors to the corresponding metallic NPs, ${ }^{30,31}$ particularly; this organic solvent has been exploited to synthesize silver NPs from silver nitrate at room temperature without using catalyst. ${ }^{31,32}$ Moreover, as aforementioned, DMF being a main solvent for PU.33 Therefore, we have exploited these observations to selfsynthesize silver NPs within PU/(THF/DMF) electrospun 


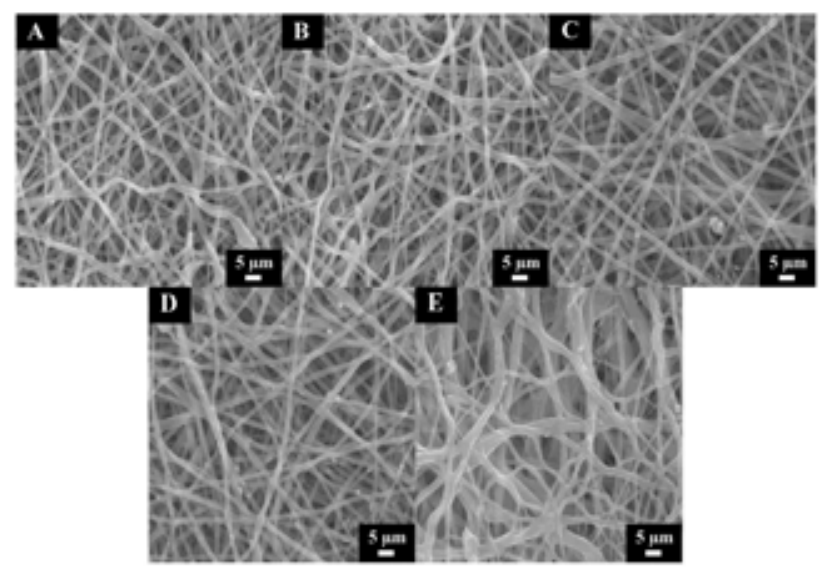

Figure 1. Scanning electron microscopy images of the PU nanofibers containing different amounts of silver nitrate, $0 \%$; (A), $2 \%$; (B), $5 \%$; (C), 7\%; (D) and 10\%; (E) silver nitrate with respect to PU.

nanofiber mats. To get full decomposition of silver nitrate into silver NPs, after electrospinning; the nanofiber mats were aged for 1 week, during this storing time; visible changes in color was observed in the electrospun mats. These mats exhibited pinkish to brown color during a period of one week; the brightness of the original and final colors was depending on the silver content in the electrospun mat, later on, no visible change in color was observed which indicated maximum decomposition of silver salt into silver metal. Figure 1 shows SEM images for all nanofiber formulations. As shown in this figure, pure $\mathrm{PU}$ and $\mathrm{PU} / \mathrm{AgNO}_{3}$ produces smooth and bead-free nanofibers. Observable NPs can be noticed with high silver nitrate contents (Figure 1, panel A, B, C, D and E).

The typical XRD patterns for pristine and PU composite nanofiber mats are presented in Figure 2. The diffraction peaks at $2 \theta$ values of $38.11,44.27,64.42$ and $77.47^{\circ}$ corresponding to (111), (200), (220), (311) crystal planes implies the persistence of crystalline silver NPs (JCDPS, card no 04-0783). As shown in Figure 2, all the standard peaks of pure silver are observed in the case of nanofibers obtained from PU/silver nitrate sol-gels. While as in case of pristine no such peaks were observed as compared with other modified counterparts. This indicates that the obtained nanofiber mats from PU/silver nitrates sol-gels do have silver nitrate in reduced form as pure silver particles and simultaneously confirming ability of DMF as reducing agent. Moreover, it can also be observed from this figure that, the intensity at (111) main plane in the standard silver crystal lattice (at $2 \theta$ values of $38.11^{\circ}$ ) increases with increasing the silver nitrate content in the original sol-gel which leads to more silver NPs in the electrospun nanofiber mats.

Figure 3 shows high magnification TEM image of one of the modified nanofiber it can be observed that NPs are present in/on the nanofiber. Overall, size of the NPs is within range

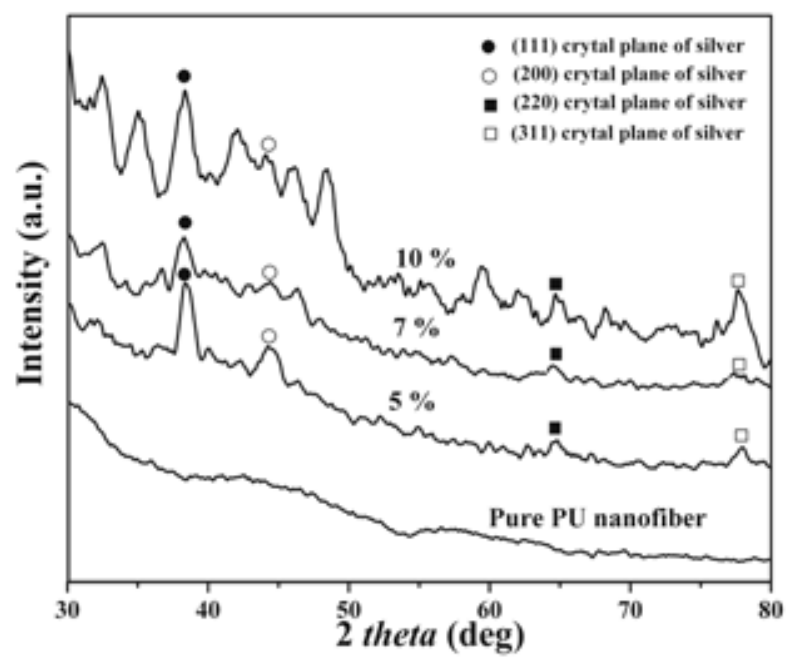

Figure 2. XRD results of the obtained nanofiber mats.

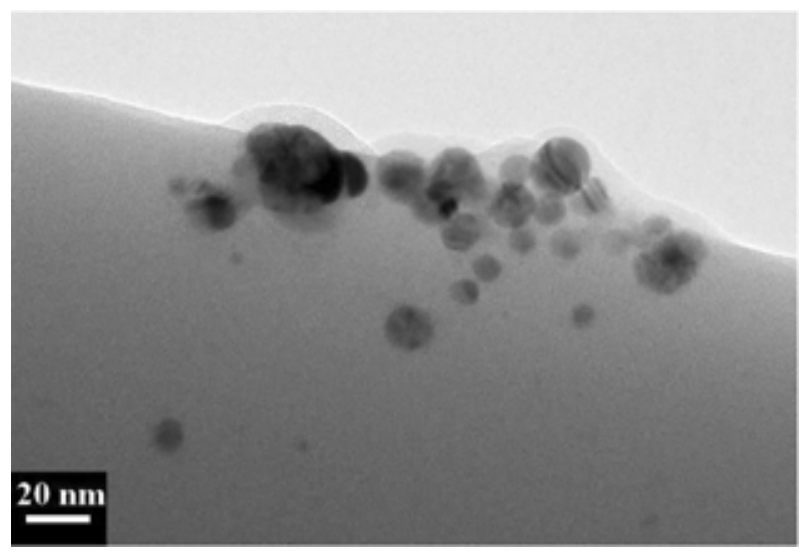

Figure 3. High magnification transmission electron micrograph of individual nanofibers showing nanoparticles.

5 to $20 \mathrm{~nm}$. In this context, Figure 4 shows the TEM images of the PU nanofibers containing different amounts of silver NPs after electrospinning. It can be observed in all the combinations ranging from 2, 5, 7 and $10 \mathrm{wt} \%$, nearly spherical NPs can be seen. It can be also noticed when TEM images are compared with SEM ones that the NPs density is higher in TEM images than SEM for all silver nitrate contents. With taken into consideration that the electron beam in TEM analysis passes through the nanofibers, so, it can detect the silver NPs incorporated inside the nanofibers, however, in a case of SEM; only the surface morphology is investigated, consequently, we can say that the number of silver NPs inside the obtained nanofibers are so many compared with the NPs synthesized on the outer surface. For instance, as shown in Figure 1(B) which reveals the nanofibers obtained from a sol-gel containing $2 \%$ silver nitrate, no NPs can be observed, however, in the corresponding TEM image (Figure 4(A)) some NPs are clearly visible (the dark dots). This observation might add good feature to the prepared nanofi- 

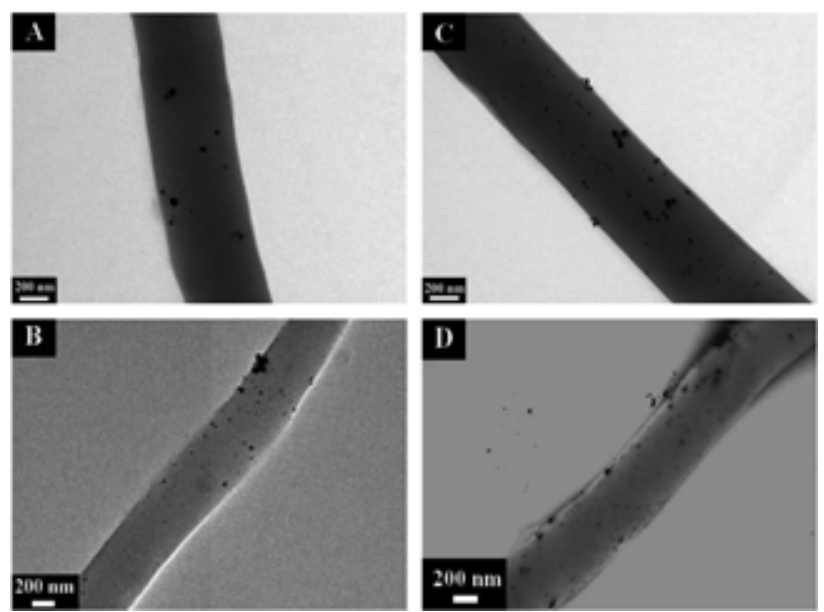

Figure 4. Transmission electron micrographs of the prepared nanofibers obtained from silver nitrate/PU sol-gels containing different amounts of silver nitrate, $2 \%$; (A), $5 \%$; (B), 7\%; (C) and 10\%; (D) silver nitrate with respect to PU.

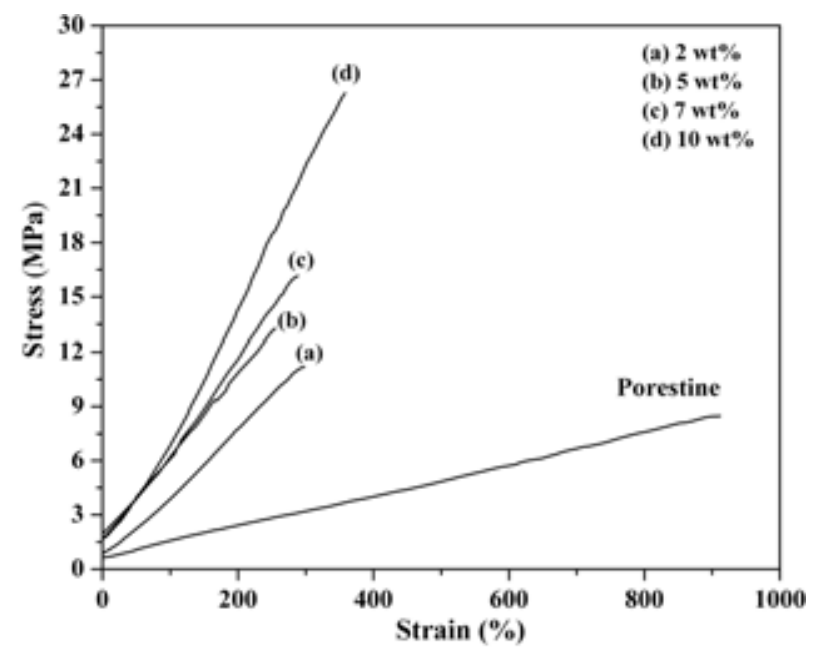

Figure 5. Stress over strain curves of the prepared silver NPs/PU nanofibers matrix obtained from silver nitrate/PU sol-gels containing different amounts of silver nitrate.

ber matrix, since some authors have been proofed that PU is biodegradable $\mathrm{e}^{34}$ so with time passing more silver NPs will appeared which enhances the antibacterial activity for the prepared nanofiber mats.

The obtained nanofibers were analyzed for the mechanical properties by following our early designed method. ${ }^{35}$ Figure 5 reveals the obtained stress-strain curves. As shown in this figure, incorporating silver NPs in PU nanofibers do have noticeable positive effect on the mechanical properties, since the fracture tensile strength increases with increasing the silver NPs content. Improving the mechanical properties of the obtained silver NPs/PU nanofibers mats strongly supports utilizing of prepared mats in many versatile applications such as filter membranes. Actually, effect of salt addition on the mechanical properties of the electrospun mats was studied in details by other researchers whom concluded that it has positive effect due to decrease the average fiber diameter and accordingly increase the total number of fibers in the electrospun mat. ${ }^{36}$

Silver has an interesting feature so-called surface plasmon resonance (SPR), this character is making the spectrophotometer more trustable to investigate silver content in the solutions. SPR is a phenomenon which occurs when light is reflected off thin metal films or NPs. A fraction of the light energy incident at a sharply defined angle can interact with the delocalised electrons in the metal surface (plasmon) thus reducing the reflected light intensity. ${ }^{37}$ In more details, when small metallic NPs are illuminated, the oscillating electric field causes the conduction electrons to oscillate coherently. In particular, silver and gold metals are the most popular materials used in this concern, ${ }^{38}$ however, silver is most commonly utilized, ${ }^{39}$ because its $d$-s band gap is in the UV region and does not damp out the plasmon mode as strongly as for gold. ${ }^{40}$ Consequently, we exploited this phenomenon to check the stability of the synthesized silver nanoparticles on the polymeric nanofibers. To be sure that the silver NPs are firmly bounded with the nanofibers and will not be lost under harsh conditions, e.g., in water purifier systems, we performed series of wash experiments. In this regard, a preweighted amount of fiber was washed with distill water at solid to liquid ratio of 3:5,000 under vigorously stirring for $25 \mathrm{~h}$. During this time period, every $2.5 \mathrm{~h}$; distilled water was replaced by fresh one. To precisely invoke spectrophotometer, the polymeric nanofibers (before and after washing) were dissolved in a proper solvent. In a case of silver NPs/PU nanofibers mats, THF has been used to dissolve the polymer and leave out silver NPs suspended in the solution. Figure 5 shows the obtained UV-Vis absorption spectra PU containing different amounts of silver NPs solutions (pristine PU/THF solution was used as blank). It is noteworthy mentioning that the weight of utilized samples was same in all silver contents and for the blank solution as well. As shown in this figure, nanofiber containing silver NPs showed absorbance band in the range of $450 \mathrm{~nm}$ and this absorbance is attributed to the characteristic surface plasmon resonance of silver NP. ${ }^{41}$ As can be also observed from Figure 6 , the absorbance intensity increases with increasing the silver content in the nanofibers. This increase is observed until $7 \%$ due to the UV instrument specification which can not detect high silver content solution. Moreover, nearly no difference between the spectra was observed for the washed and unwashed mats. To practically demonstrate the results, Table I shows the absorbance intensities at $450 \mathrm{~nm}$ for the solutions of the as-prepared and after washing process; also the relative differences were calculated. As shown in this table, the relative difference is very small even for the high silver content samples. According to these results, it can be concluded that silver NPs are still remaining in the washed 


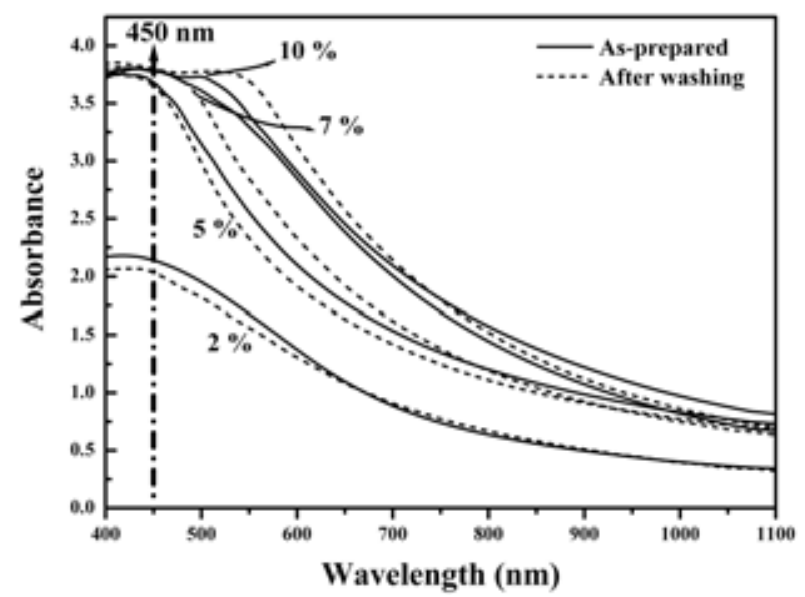

Figure 6. UV-visible spectroscopy of silver NPs/PU nanofibers matrix obtained from sol-gels containing different amounts of silver nitrates; as-prepared (solid lines) and after washing with water (doted lines).

Table I. Absorbance Intensities and Relative Differences at 450 nm for the As-Prepared and Washed Silver-Doped PU Nanofiber Mats

\begin{tabular}{cccc}
\hline $\begin{array}{c}\text { Silver Content, } \\
\%\end{array}$ & As-prepared & After Washing & $\begin{array}{c}\text { Relative } \\
\text { Difference }\end{array}$ \\
\hline $2 \%$ & 2.14187 & 2.01931 & 0.05722 \\
$5 \%$ & 3.81406 & 3.60796 & 0.05404 \\
$7 \%$ & 3.95978 & 3.73338 & 0.05717 \\
$10 \%$ & 3.80306 & 3.77040 & 0.00858 \\
\hline
\end{tabular}

nanofibers, almost no losses in the silver NPs had taken place. These results support TEM analyses (Figure 3 and Figure 4) indicating that most of the NPs are imprisoned inside the nanofibers. Moreover, these results reveal that even the NPs present on the surface are well attached with the nanofibers since no change in the absorbance intensity even for the high silver NPs content nanofibers which contain many NPs on the outer surfaces. Therefore, one can recommend these nanofibers for long term contact with water operations; like antimicrobial water filters.

For filtering application, there should be sufficient amount of void space inside the nanofiber mats in the form of pores.
The water permeability of pure PU and modified counterparts were analyzed for porosity investigation. The data are shown in Table II. As shown in this table, adding of silver nitrate into PU sol-gel has distinct influence on porosity of nanofiber mats. All the measured parameters indicating the porosity (i.e. porosity and average pore diameter, area and volume) increase upon increasing the silver content in the electrospun nanofibers. Accordingly, the size of the nanofiber and pore size of the nanofiber mats might be controlled by changing silver nitrate content during the electrospinning process. Overall, this result supports utilizing of the prepared $\mathrm{Ag} / \mathrm{PU}$ nanofiber mats in water purifier systems.

As far as antimicrobial agents are considered, silver is considered material of choice, it is also established that silver enhances the epithilization. ${ }^{5,42}$ Silver and its forms are potent antimicrobials against various bacterial species. The exact mechanism how silver participates in distortion of bacteria is unfortunately still unclear. However, generally it is believed that sliver mainly makes denaturation and oxidization for cell organelles which lead to suppress the cell division. It is well known fact when a critical amount of antibacterial compound (capable of inhibiting microbial growth) is in contact with bacterial strains the clear area near that contact is formed and these areas are referred as zones of inhibition. ${ }^{43}$ Figure 7 shows $12 \mathrm{~h}$ incubated petriplates of E. coli and S. typhimurium grown in presence of

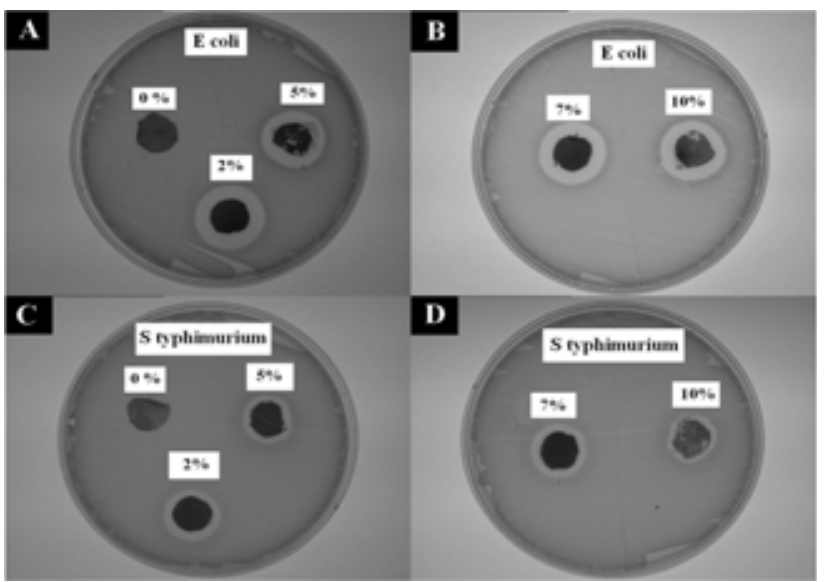

Figure 7. Agar plates showing zones of inhibitions after $12 \mathrm{~h}$ of incubation for two different types of bacteria; E. coli (A and B) and S. typhimurium (C and D).

Table II. Porosity Test of the Obtained Nanofibers

\begin{tabular}{ccccc}
\hline $\mathrm{PU} / \mathrm{AgNO}_{3}$ & Average Pore Diameter $(\mu \mathrm{m})$ & Average Pore Volume $\left(\mu \mathrm{m}^{3}\right)$ & Average Pore Area $\left(\mu \mathrm{m}^{2}\right)$ & Average Porosity $(\%)$ \\
\hline $10 \%$ & 5.5 & 4.9 & 3 & 99 \\
$7 \%$ & 5.5 & 3.9 & 2.5 & 97 \\
$5 \%$ & 5.4 & 3.9 & 2.1 & 90 \\
$2 \%$ & 4.8 & 3.5 & 1.8 & 78 \\
Pure PU & 4.2 & 2.7 & 1 & 70 \\
\hline
\end{tabular}


circular nanofiber disk. From this figure we can clearly observe the ZoI around the circular nanofiber disks containing different percentages of silver $(2,5,7$ and $10 \%)$, while as in case of pure PU $(0 \%)$ nanofiber these $\mathrm{ZoI}$ were missing. Formation of ZoI around the nanofiber disks is an indicator for inhibition of bacterial colonies near the vicinity of nanofiber disk edges. Basically, due to presence of silver in the nanofiber disk, it may lead to kill the microorganism around the disk which results in formation of clear circular zones. Generally, it is considered that diameter of the inhibited area is a measure of the effectiveness or resistance of the bacteria towards organic antimicrobial agent. However, in case silver a previous work has concluded that zone of inhibition is only qualitative and provides no means of assessing quantities of silver that have been released. ${ }^{43}$ Therefore, diameter of a zone is not in itself sufficient to determine efficacy of silver and its forms. Accordingly, we did not find any significant difference with varying of silver content in the nanofiber mats.

To investigate the influence of silver NPs on the living bacterial cells, the nanofiber mats (containing 0 and $10 \%$ silver) were interacted with a cell suspension (density of $1 \times 10^{7}$ cells $/ \mathrm{mL}$ in PBS) for $5 \mathrm{~h}$. Figure 8 shows the obtained results. As shown in this figure, morphological changes are observed. A significant surface changes occurred for the bacteria incubated in presence of silver NPs/PU nanofibers than in case of pure PU nanofibers. It can be also seen that distorted porous membranes (pits) on bacterial strains which exactly matches with a previously reported work. ${ }^{44}$

The colony forming units is an excellent demonstration to find out the antimicrobial nature of any compound. As can be indicated form Figure 9 which shows the data obtained from CFU results, the prepared silver NPs/PU nanofiber mats can be considered as effective and trustable antibacterial agent. As aforementioned, after culturing the $E$. coli and

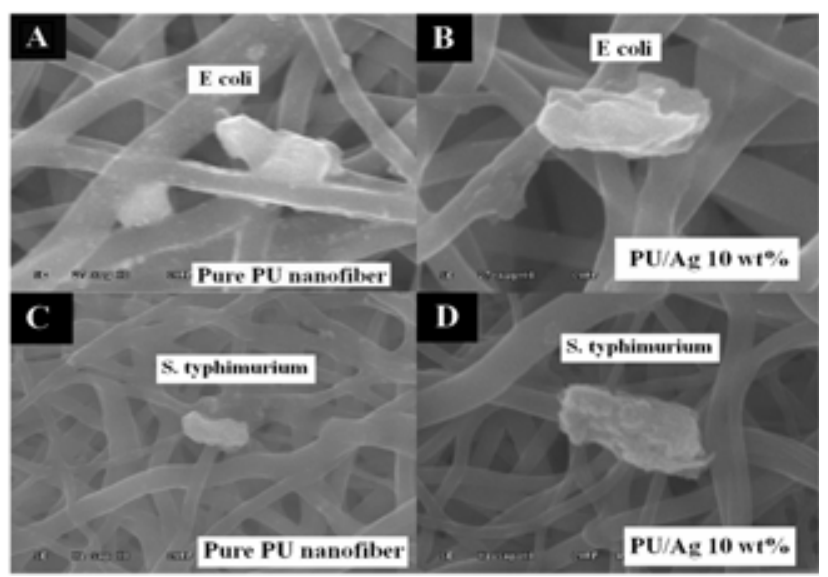

Figure 8. Bio-SEM images showing morphological changes upon interaction between silver-free nanofibers and nanofibers obtained from sol-gel contains $10 \%$ silver nitrate with two different types of bacteria; E. coli (A and B) and S. typhimurium (C and D).

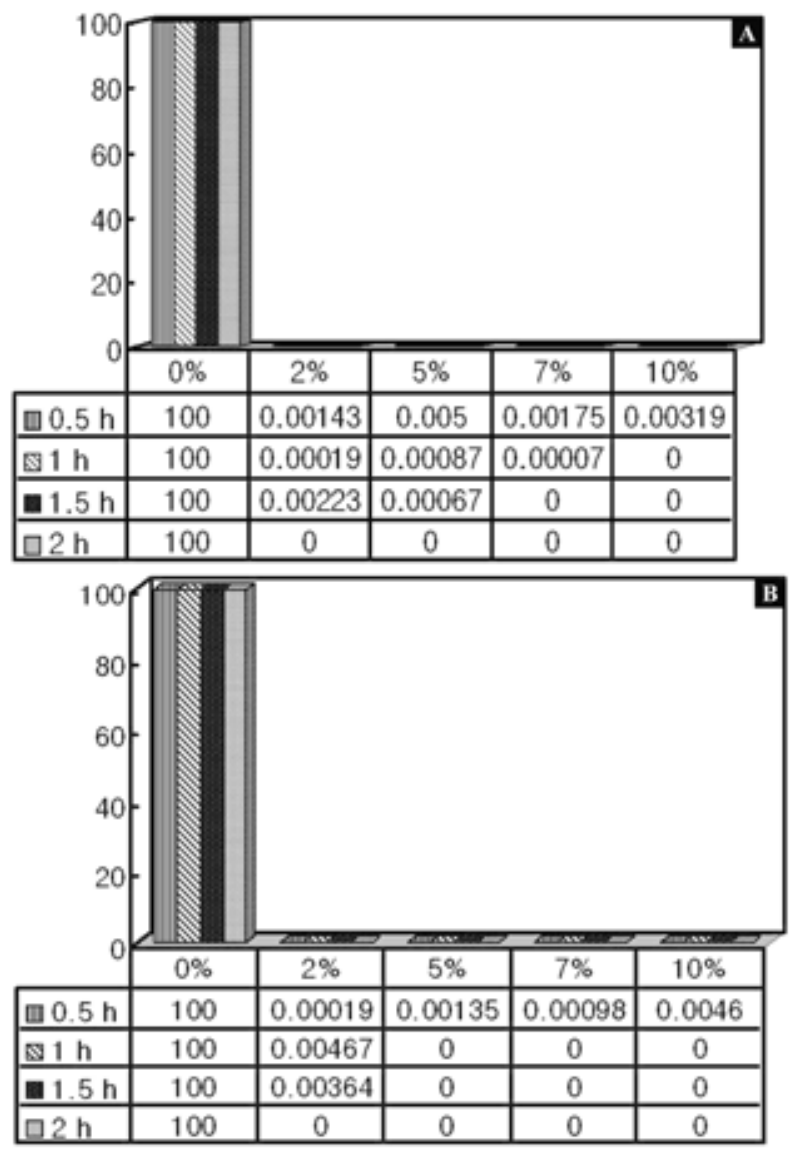

Figure 9. CFU results obtained by growing of $E$. coli (A) and $S$. typhimurium, (B) in presence of pure PU nanofibers and nanofibers obtained from sol-gels have different amounts of silver nitrate. The tables represent the numeric results in each case.

S. typhimurium with the cell density of $1 \times 10^{7}$ in nutrient broth for the period of 30, 60, 90 and 120 min of incubation time. The microbial colonies raised after incubation in presence of pure and different grades of silver containing nanofibers were counted. It can be seen in Figure 9(A) and (B), the nanofiber containing zero percent of silver shows $100 \%$ microbial growth the reason for this that this formulation does not have any silver content, so, full growth of microbial population was seen which is quite obvious. While as, its counter parts which contains loaded amount of silver shows drastic decrease in cell population with respect with time and amount of silver content in them as seen in the Figure 9(A) and (B), (see the tables under the figures). Overall, these results indicate that the nanofibers containing silver NPs reveal well response with the bacteria, they are sufficient to kill almost all the bacteria in the cited times in Figure 9. It is noteworthy mentioning that the colonies formed from pristine nanofibers which contains $0 \%$ silver, were numerous and were set as 100 cells and other values were compared to this value. Overall, results generously 
indicate well establishing facts of using these antimicrobial PU nanofibers containing silver NPs as desired candidate for future wound healing agents and as for antibacterial filters for water purifier systems.

\section{Conclusions}

Nano-biotechnologically engineered silver nitrate/PU solgel can be easily electrospun to form smooth nanofibers. However, presence of DMF can result in reduce $\mathrm{AgNO}_{3}$ to silver NPs in simple and biologically safe way. Moreover, the obtained silver NPs do have strong stagnancy since these NPs have not been released after many successive washing processes of the nanofiber mats by big amount of fresh water for long time. This observation supports the recommendation of utilizing the introduced nanofiber mats in water purifying systems especially incorporation of silver NPs does have positive impact of the mechanical properties of the electrospun nanofiber mats. The antimicrobial test has been conducted to ensure that the obtained nanofibers can be used as antimicrobial wound dressing materials; the obtained results were satisfactory. Avoiding using any foreign reducing chemicals, well stability of the NPs open a new avenue for the prepared nanofibers to be used in various biomedical applications.

Acknowledgement. This work was supported by the grant of the Korean Ministry of Education, Science and Technology (The Regional Core Research Program/Center for Healthcare Technology \& Development, Chonbuk National University, Jeonju 561-756 Republic of Korea). Author is thankful to KBSI, Jeonju branch for providing facility to perform porosity tests.

\section{References}

(1) A. L. Peter, Adv. Drug Deliver. Rev., 57, 1471 (2005).

(2) T. J. Berger, J. A. Spadaro, S. E. Chapin, and R. O. Becker, Antimicrob. Agents Chemother., 9, 357 (1976).

(3) S. Silver, FEMS Microbiol. Rev., 27, 341 (2003).

(4) L. S. Nair and C. T. Laurencin, J. Biomed. Nanotech., 3, 16 (2007).

(5) J. Tian, K. K. Wong, C. M. Ho, C. N. Lok, W. Y. Yu, C. M. Che, J. F. Chiu, and P. K. Tam, Chem. Med. Chem., 2, 129 (2007).

(6) J. Trogolo, Filtr. Sep., 43, 28 (2006).

(7) M. E. Innes, N. Umraw, J. S. Fish, M. Gomez, and R. C. Cartotto, Burns, 27, 621 (2001).

(8) M. A. Butkus, M. Talbot, and M. P. Labare, Water Res., 39, 4925 (2005).

(9) R. F. DeBono, A. Helluy, M. Heimlich, and U. J. Krull, Sens. Actuators, B, 11, 487 (1993).

(10) P. W. Gibson, H. L. Schreuder-Gibson, and D. Rivin, AlChE J., 45, 190 (1999).

(11) P. W. Gibson, H. L. Schreuder-Gibson, and D. Rivin, Colloid
Surf. A, 187/188, 469 (2001).

(12) W. K. Son, J. H. Youk, and W. H. Park, Carbohydr. Polym., 65, 430 (2006).

(13) H. K. Lee, E. H. Jeong, C. K. Chi Baek, and J. H. Youk, Mater. Lett., 59, 2977 (2005).

(14) Y. Y. Duan, J. Jia, S.-H Wang, Sh. W. Yan, L. Jin, and Z. Y. Wang, J. Appl. Polym. Sci., 106, 1208 (2007).

(15) H. Kong and J. Jang, Langmuir, 24, 2051 (2008).

(16) K. H. Hong, J. L. Park, I. H. Sul, J. H. Youk, and T. J. Kang, J. Polym. Sci. Part B: Polym. Phys., 44, 2468 (2006).

(17) Q. Zhang, D. Wu, S. Qi, Z. Wu, X. Yang, and R. Jin, Mater. Lett., 61, 4027 (2007).

(18) Y. H. Jung, H. Y. Kim, D. R. Lee, S. Y. Park, and M. S. Khil, Macromol. Res., 13, 385 (2005).

(19) J. Doshi and D. H. Reneker, J. Electrostat., 35, 151 (1995).

(20) H.-J. Jin, M.-O. Hwang, J. S. Yoon, K. H. Lee, I.-J. Chin, and M.-N. Kim, Macromol. Res., 13, 73 (2005).

(21) C. P. Barnes, S. A. Sell, E. D. Boland, D. G. Simpson, and G. L. Bowlin, Adv. Drug Deliver. Rev., 59, 1413 (2007).

(22) D. Aussawasathien, C. Teerawattananon, and A. Vongachariy, J. Membr. Sci., 315, 11 (2008).

(23) S. Kidoaki, I. K. Kwon, and T. Matsuda, J. Biomed. Mater. Res. Part B: Appl. Biomater., 786, 219 (2006).

(24) T. K. Khlystalova, M. N. Kurganova, A. I. Demina, M. B. Petova, and O. G. Tarakanov, Mech. Compos. Mater., 21, 763 (1986).

(25) J. H. Han, J. D. Taylor, D. S. Kim, Y. S. Kim, Y. T. Kim, G. S. Cha, and H. Nam, Sens. Actuators B, 123, 384 (2007).

(26) N. Hains, V. Friscic, and D. Gordos, Int. J. Cloth. Sci. Technol., 15, 250 (2003).

(27) M. S. Khil, D. I. Cha, H. Y. Kim, I. S. Kim, and N. Bhattarai, J. Biomed. Mater. Res. B: Appl. Biomater, 67, 657 (2003).

(28) C. Yao, X. Li, K. G. Neoh, Z. Shi, and E. T. Kang, J. Membr Sci., 320, 295 (2008).

(29) E. H. Jeong, J. Yang, and J. H. Youk, Mater. Lett., 61, 3991 (2007).

(30) I. Pastoriza-Santos and L. M. Liz-Marzan, Pure Appl. Chem., 72, 83 (2000).

(31) I. Pastoriza-Santos and L. M. Liz-Marzan, Langmuir, 15, 849 (1999).

(32) N. L. Lala, R. Ramaseshan, L. Bojun, S. Sundarrajan, R. S. Barhate, L. Ying-Jun, and S. Ramakrishna, Biotechnol. Bioeng., 97, 1357 (2007).

(33) H. Zhuo, J. Hu, S. Chen, and L. Yeung, J. Appl. Polym. Sci., 109, 406 (2008).

(34) S. A. Guelcher, Tissue Eng. Part B, 14, 3 (2008).

(35) J. H. Park, B. S. Kim, Y. C. Yoo, M. S. Khil, and H. Y. Kim, J. Appl. Polym. Sci., 107, 2211 (2008).

(36) K. W. Kim, Effects of Electrospinning Parameters on the Fiber Formation, PhD theses, Chonbuk Nat. Univ. (2007), and reference therein.

(37) C. F. Bohrem and D. R. Huffman, Absorption and Scattering of Light by Small Particles, Wiley-Interscience, New York, 1983.

(38) T. Nikolajsen, K. Leosson, and S. I. Bozhevolnyi, Appl. Phys. Lett., 85, 5833 (2004).

(39) C. L. Haynes and R. P. Van Duyne, J. Phys. Chem. B, 107, 7426 (2003).

(40) H. H. Jose, I. Martini, and V. H. Gregory, J. Phys. Chem. B, 
F. A. Sheikh et al.

102, 6958 (1998).

(41) K. Patel, S. Kapoor, D. P. Dave, and T. Mukerjee, J. Chem. Sci., 117, 53 (2005).

(42) B. S. Atiyeh, M. Costagliola, S. N. Hayek, and S. A. Dibo, Burns, 33, 139 (2007).
(43) C. L. Gallant-Behm, H. Q. Yin, S. Liu, J. P. Heggers, R. E. Langford, M. E. Olson, D. A. Hart, and R. E. Burrell, Wound Repair Regen., 13, 412 (2005).

(44) K. H. Choa, J. E. Park, T. Osaka, and S. G. Park, Electrochim. Acta, 51, 956 (2005). 